\title{
LETTER \\ Delay Analysis of Car-to-Car Reliable Data Delivery Strategies Based on Data Mulling with Network Coding
}

\author{
Joon-Sang PARK ${ }^{\dagger * a)}$, Uichin LEE $^{\dagger \dagger}$, Soon Young OH $^{\dagger \dagger}$, Mario GERLA ${ }^{\dagger \dagger}$, \\ Desmond Siumen LUN ${ }^{\dagger \dagger}$, Nonmembers, Won Woo RO ${ }^{\dagger \dagger \dagger}$, and Joonseok PARK ${ }^{\dagger \dagger \dagger \dagger}$, Members
}

\begin{abstract}
SUMMARY Vehicular ad hoc networks (VANET) aims to enhance vehicle navigation safety by providing an early warning system: any chance of accidents is informed through the wireless communication between vehicles. For the warning system to work, it is crucial that safety messages be reliably delivered to the target vehicles in a timely manner and thus reliable and timely data dissemination service is the key building block of VANET. Data mulling technique combined with three strategies, network codeing, erasure coding and repetition coding, is proposed for the reliable and timely data dissemination service. Particularly, vehicles in the opposite direction on a highway are exploited as data mules, mobile nodes physically delivering data to destinations, to overcome intermittent network connectivity cause by sparse vehicle traffic. Using analytic models, we show that in such a highway data mulling scenario the network coding based strategy outperforms erasure coding and repetition based strategies.

key words: network coding, VANET, data mule, analysis
\end{abstract}

\section{Introduction}

Wireless networks for automobiles, i.e., vehicular ad hoc networks (VANET), are an emerging technology mainly developed to provide more safety to the car drivers and the passengers. The technique aims to reduce the risk of automobile accidents by providing an early collision warning system. Any chance of car crash can be informed and avoided beforehand through the wireless communication between vehicles. For instance, suppose that a critical traffic/safety situation occurs on a highway; in this case, the safety warning message could be triggered on one or more lead cars and propagated to vehicles following several miles behind.

Certainly, VANET will be equipped with advanced radios and wireless LAN grade bandwidth, thus allowing aggressive data rates and applications. Although simple text data can still deliver a necessary warning message, more comprehensive information carried via multimedia such as video can help drivers to make more accurate decision and several multimedia data delivery schemes have been proposed for vehicular environments [4], [5], [10]. For the

\footnotetext{
Manuscript received March 24, 2008.

Manuscript revised June 21, 2008.

${ }^{\dagger}$ The author is with Hongik University, Korea.

${ }^{\dagger}$ The authors are with University of California, Los Angeles, USA.

${ }^{+\dagger}$ The author is with Massachusetts Institute of Technology, USA.

${ }^{\dagger+\dagger}$ The author is with Yonsei University, Korea.

${ }^{+1+\dagger}$ The author is with Inha University, Korea.

${ }^{*}$ Corresponding author.

a) E-mail: jsp@ hongik.ac.kr

DOI: 10.1093/ietisy/e91-d.10.2524
}

above warning systems to work, however, it is crucial that safety related messages be reliably delivered to all the impacted nodes in VANET in a timely manner and thus reliable and timely data dissemination service is the key building block of navigation safety applications in VANET.

There is a vast literature on reliable data dissemination in VANET and more generally in ad hoc networks (e.g., [7], [11], [12]). The main focus however has been on short alert messages in fully connected VANET. The short message application in fully connected VANET can be supported by conventional solutions such as repetition of messages. The reliable data dissemination problem becomes much more complex with multimedia data since multimedia files are innately large. When disseminated without proper controls, they can cause severe network congestion and/or intolerable data delivery delay. Here, advance coding techniques such as network coding can play a critical role. Advance coding techniques can provide reliability with reduced delivery delay compared to a simple coding technique such as repetition.

The service must also be robust to the unpredictable nature of VANET. Cars may become separated on the highway, forming platoons. If an accident occurs, a platoon that follows by 30 seconds, say, will automatically incur a 30 second delay. To make this delay shorter we can exploit vehicles coming in the opposite direction (assuming that the highway has multiple lanes in both directions). This approach uses the vehicles in the opposing directions as "data mules." In fact, as shown in [13] it is better to employ multiple vehicles in parallel (when available) to deliver a relatively large multimedia file using the data mule approach.

In this letter, we analyze the performance of reliable data delivery strategies in intermittently connected vehicular networks such as the highway scenario described above. We compare network coding, erasure coding, and the simple repetition based strategies and show that the network coding based strategy outperforms erasure coding and repetition based strategies in the highway scenario. By network coding generally attributed to Ahlswede et al. [1], we refer to the notion of performing coding operations on the contents of packets throughout a network. In particular, the network coding method we assume in the comparison is so-called random linear coding proposed in [2], [6].

The rest of this letter is organized as follows. The three strategies are compared via an mathematical analysis in Sects. 2 and 3 concludes this letter. 


\section{Analysis}

In this section we analyze the performance of the delivery models with opposite direction vehicles that act as "data mules." We are interested in evaluating different schemes and their delayed delivery performance. Figure 1 shows the overall diagram of short segments of a certain freeway.

Let $P_{f}-k$ and $P_{r}-k$ denote the $k$-th platoon in a forward and reverse direction respectively. In our specific scenario, the vehicles exchange information about an accident. Namely, vehicles located just in front of the accident site multicast video streams to the platoon $P_{f}-1$. Obviously the data cannot propagate immediately to other disconnected platoons such as $P_{f}-2$. Our freeway relay model uses platoons in the opposite direction to pick up, carry, and forward the data to the other platoons on the forward direction, e.g., $P_{f}-2$.

We are interested in calculating the delay of delivering the complete data file to the other platoons. This can be simply expressed as follows:

$$
\text { Delay }=\min (\text { overtake delay, relay delay })
$$

Overtake delay is defined as the time for a random platoon to catch up with (reach within its communication range) the source platoon (or other platoons that have merged into the source platoon) that is driving in the same direction. Relay delay is defined as the time for a target platoon to receive the whole data from data mule platoons driving in the opposite direction. For example, let us say that we have a target platoon $P_{f}-2$ as shown Fig. 1. Overtaking delay is the time for $P_{f}-2$ to catch up with the source platoon $P_{f}-1$ and relay delay is the time for $P_{r}-1$ to encounter $P_{f}-2$. Given the limited data transfer rate between two crossing platoons, the target platoon may have to receive different parts of the data file from different platoons, i.e. data mules. This is the main focus of our analysis.

When relaying data, we could image the following strategies:

- Relay without coding $(R-W C)$ - A platoon passing by the accident site randomly picks up a number of packets and "data mules" them to the disconnected target platoon.

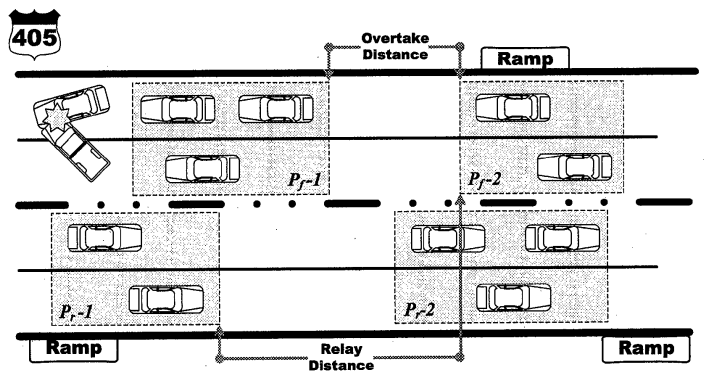

Fig. 1 Highway relay model scenario.
- Relay with erasure coding (R-EC) - A source encodes the data using erasure coding. Erasure coding protects from packet loss caused by relaying platoons that may exit the highway.

- Relay with network coding $(R-N C)$ - The data is distributed using random linear network coding.

Vehicles arrive at independently distributed random intervals. In the traffic theory [8], this type of random arrival is often modeled using an exponential distribution with parameter $\lambda$ where $\lambda$ is the flow in vehicles/second. Without loss of generality this can be extended also to a platoon arriving at the scene of the accident on the highway. The size of a random platoon could be represented as a geometric distribution with mean $s_{p}$. Then, the overall arriving process is simply modeled as a compound Poisson process. Since our focus is calculating the relay delay, we simply assume that the speeds of platoons are constant with $v_{0}$. The distance between two platoons are purely determined by the underlying Poisson arrival process.

Vehicles within a platoon may leave the highway. This depends on the density of ramps along the highway as well as the probability of defecting from a given platoon. Let $d_{r}$ denote the density of ramps and $\bar{p}_{l}$ denote the average defecting probability from a given platoon. ${ }^{\dagger}$ The ineffectiveness of a random platoon in terms of delivering packets can be expressed $p_{i e} \propto d_{r} \cdot \bar{p}_{l}$, thus $p_{i e}=\alpha \cdot d_{r} \cdot \bar{p}_{l}$ where $\alpha$ is a constant. Let $p_{e}$ denote the effectiveness of a random platoon, which is given as $p_{e}=1-p_{i e}$. Let $N_{p}(t)$ denote the number of packets that a random platoon can pick up at time $t$; for example, in the figure how many packets $P_{r^{-}}-1$ can pick up from $P_{f}-1 .^{\dagger \dagger}$ For ease of analysis, we assume that a platoon can pick up on average $\bar{N}_{p}$ packets. The effective number of packets delivered to the target platoon is simply given as $p_{e} \bar{N}_{p}$.

Let $N_{d}$ denote the total number of packets for a given data file that must be delivered. We assume that $N_{d}>p_{e} \bar{N}_{p}$, or we need multiple number of platoons to get the whole data. Since a random platoon arrives at the highway with a Poisson process, the average delay between two platoons is simply given as $\bar{T}_{h}=1 / \lambda$. From this, we see that the average distance is simply $\bar{D}_{h}=\bar{T}_{h} \times v_{0}$. We model a low traffic flow scenario such that the average distance is larger than the communication range, i.e., two consecutive platoons cannot directly communicate. Now we are ready to analyze the proposed schemes.

Relay without coding - Each "mule" platoon randomly picks up $\bar{N}_{p}$ packets and delivers on average $p_{e} \bar{N}_{p}$ packets to the target platoon. This problem is analogous with the coupon collection problem. The number of coupons, i.e., the number of data packets that we need to deliver, is given as $N_{d}$. At each step, we draw a coupon whose type is uni-

${ }^{\dagger}$ The defecting probability mainly depends on the size of a platoon.

${ }^{\dagger \dagger} N_{p}(t)$ is directly related to the length of a platoon as well as the speed of a platoon. 
formly distributed among all $N_{d}$ types. Thus, the average number of trials required at each step to get a new coupon increases as more coupons have been collected. Let $T_{N_{d}}$ be the time by which we have collected coupons belonging to all $N_{d}$ distinct types. Then, the expected number of trials that we need to make is simply given as:

$$
\begin{aligned}
\mathbb{E}\left[T_{N_{d}}^{R-W C}\right] & =1+\frac{N_{d}}{N_{d}-1}+\cdots+\frac{N_{d}}{1} \\
& =N_{d} \sum_{k=1}^{N_{d}} \frac{1}{k} \\
& \simeq N_{d} \ln N_{d}
\end{aligned}
$$

Recall now that each platoon can carry $p_{e} \bar{N}_{p}$ number of packets, and thus, the expected number of platoons that a target platoon must see (i.e., passes the data mule can make in order to transfer the file) is given as $\left[\mathbb{E}\left[T_{N_{d}}^{R-W C}\right] / p_{e} \bar{N}_{p}\right]$. It is interesting to note that since the target platoon travels in an opposite direction, the expected delay for the target platoon to meet a random platoon is simply given as $\bar{T}_{h} / 2$. Therefore, the overall delay $D_{R-W C}$ is given as

$$
\begin{aligned}
D_{R-W C} & =\left\lceil\frac{\mathbb{E}\left[T_{N_{d}}^{R-W C}\right]}{p_{e} \bar{N}_{p}}\right\rceil \times \frac{\bar{T}_{h}}{2} \\
& =\left\lceil\frac{N_{d} \ln N_{d}}{p_{e} \bar{N}_{p}}\right\rceil \times \frac{\bar{T}_{h}}{2}
\end{aligned}
$$

Thus, the time grows with $N_{d} \ln N_{d}$, where $N_{d}$ is the data file size.

Relay with erasure coding - Given that redundancy factor is $r(>0)$, erasure coding produces $N_{d}(1+r)$ coded packets where $N_{d}$ is the number of the original data packets. The key property of erasure coding is that the original data can be reconstructed from any of $N_{d}$ packets. R-EC is quite similar to R-WC but we now only collect any subset with size $N_{d}$ of $N_{d}(1+r)$ coded packets; then, we may want to ask what the relative advantage of using erasure coding in terms of the average delay. Similar to R-WC, the expected number of trials for R-EC is given as:

$$
\begin{aligned}
\mathbb{E}\left[T_{N_{d}}^{R-E C}\right] & =1+\frac{N_{d}(1+r)}{N_{d}(1+r)-1}+\cdots+\frac{N_{d}(1+r)}{N_{d} r} \\
& =N_{d}(1+r) \sum_{k=N_{d} r}^{N_{d}(1+r)} \frac{1}{k} \\
& \simeq N_{d}(1+r) \ln \left(1+\frac{1}{r}\right)
\end{aligned}
$$

Lemma 2 in Appendix shows the proof of the approximation used in Eq. (8). The overall delay $D_{R-E C}$ is given as

$$
D_{R-E C}=\left\lceil\frac{N_{d}(1+r) \ln (1+1 / r)}{p_{e} \bar{N}_{p}}\right\rceil \times \frac{\bar{T}_{h}}{2}
$$

Note that the constant logarithm factor $\ln (1+1 / r)$ plays a key role determining $\mathbb{E}\left[T_{N_{d}}^{R-E C}\right]$. If $r \geq 0.5$, we have $\mathbb{E}\left[T_{N_{d}}^{R-E C}\right] \simeq$
$N_{d}(1+r)$. On the other hand given that

$$
r=\frac{-\left(1-\frac{1}{N_{d}}\right)+\sqrt{\left(1-\frac{1}{N_{d}}\right)^{2}+\frac{4}{N_{d}}}}{2}
$$

the resulting equation is exactly the same as $\mathbb{E}\left[T_{N_{d}}^{R-W C}\right]$ with $N_{d}(1+r)$ original data packets. It is important to note that the delay improvement of R-EC comes at the cost of increased redundancy factor $r$. The video source has to generate and broadcast redundant packets proportional to the total number of packets $\left(r \times N_{d}\right)$; thus, we are utilizing the channel less efficiently (also potentially causing more collisions).

Relay with network coding - Both R-WC and R-EC are analogous to coupon collection. The intrinsic problem of coupon collection is that once we have collected half of the coupons, it takes a progressively longer and longer time to collect the rest of coupons. On the other hand, "algebraic mixing" of the original data in random network coding help us to attain near optimal bound of data dissemination. The key idea of random network coding is that no matter how many coded packets you have collected so far, any new random coded packet is "helpful" with high probability. This claim has proven in [3], and for the sake of completeness, we include the result in Lemma 1. Suppose that we use random linear coding with a finite field of size $q$. As mentioned earlier, a coded packet includes a code-vector as well as coded data. In order to decode packets, one must collect $N_{d}$ independent code vectors (i.e., rank $N_{d}$ ).

Lemma 1: Suppose node $v$ transmits a coded packet to node $u$. Let $S_{u}^{-}$and $S_{v}^{-}$denote the subspaces spanned by the code-vectors with $u$ and $v$ respectively at the beginning. Let $S_{u}^{+}$denote the subspaces spanned by the code-vectors by $u$ after receiving a coded packet from $v$. Then,

$$
\operatorname{Pr}\left(\operatorname{dim}\left(S_{u}^{+}\right)>\operatorname{dim}\left(S_{u}^{-}\right) \mid S_{v}^{-} \nsubseteq S_{u}^{-}\right) \geq 1-\frac{1}{q},
$$

where $q$ is the size of the field.

Let $\tilde{T}_{N_{d}}^{R-N C}$ denote the number of IID Bernoulli trials with success probability $p=1-1 / q$. Trials with probability $p$ are being continued until the $N_{d}$-th success. The distribution gives the probability that $z$ experiments are needed to reach $N_{d}$ successes, which is known as a negative binomial distribution as shown below.

$$
\operatorname{Pr}\left(\tilde{T}_{N_{d}}^{R-N C}=z\right)=\left(\begin{array}{c}
z-1 \\
N_{d}-1
\end{array}\right) p^{N_{d}}(1-p)^{z-N_{d}},
$$

where $z=N_{d}, N_{d}+1, \cdots$. Thus, the expected number of trials is given as

$$
\mathbb{E}\left[\tilde{T}_{N_{d}}^{R-N C}\right]=\frac{N_{d}}{p}=N_{d} \frac{q}{q-1}
$$

From Lemma 1, the actual success probability is greater than $p$, and thus, $\mathbb{E}\left[\tilde{T}_{N_{d}}^{R-N C}\right]$ will be the upper bound of the mean number of trials: 


$$
N_{d}<\mathbb{E}\left[T_{N_{d}}^{R-N C}\right] \leq \mathbb{E}\left[\tilde{T}_{N_{d}}^{R-N C}\right]
$$

As the field size increases, Eq. (12) shows that the upper bound approaches to the lower bound. For a large field size, ${ }^{\dagger}$ we conclude that

$$
\mathbb{E}\left[T_{N_{d}}^{R-N C}\right] \simeq N_{d}
$$

Therefore, the overall delay $D_{R-N C}$ is given as

$$
D_{R-N C}=\left\lceil\frac{N_{d}}{p_{e} \bar{N}_{p}}\right\rceil \times \frac{\bar{T}_{h}}{2}
$$

Note that compared to R-WC where the delivery time is proportional to $N_{d} \ln N_{d}$, that of network coding is proportional to $N_{d}$. R-EC can achieve the same delay, but again with great cost. In conclusion, network coding provides a considerable improvement in the time required to propagate reliably via "data muling" critical video streams to disconnected platoons.

Network coding's overhead exists in different types: code construction delay, encoding/decoding delay, and, especially for the random network coding we assume in this letter, a larger packet size. One approach proposed in [2] suggests that each packet carry a so-called encoding vector. This means that nodes transmit larger-size packets. However, as pointed out in [2], the overhead is small, around $3 \%$, and a larger packet size does not always contribute to a lower packet transmission rate since the transmission rate depends far more on the wireless communications technology than a small variance in the packet size. Also, there are other coding techniques that limit the impact of the overhead of network coding to the performance such as one proposed in [9]. Thus, we ignore the overhead of network coding in our analysis.

\section{Conclusions}

In this letter, we explored the problem of "delayed" delivering of large data to disconnected platoons on a highway using the vehicles traveling in the opposite direction as "data mules." Considering the fact that the opposite vehicles can randomly pick up (by "osmosis") only a limited number of the packets at each pass, this problem can be reformulated as a "coupon collector" problem. Using simple analytic models, we have shown that Network Coding helps reducing data delivery relay in such a highway scenario.

\section{Acknowledgement}

Joon-Sang Park's work was supported by the Hongik University new faculty research support fund.

\section{References}

[1] R. Ahlswede, N. Cai, S.-Y.R. Li, and R.W. Yeung, "Network information flow," IEEE Trans. Inf. Theory, vol.46, no.4, pp.1204-1216, July 2000.

\footnotetext{
${ }^{\dagger}$ A common $q$ value used in real NC implementations is $2^{16}$.
}

[2] P. Chou, Y. Wu, and K. Jain, "Practical network coding," Proc. 51st Allerton Conf. Communication, Control and Computing, 2003.

[3] S. Deb, M. Médard, and C. Chout, "Algebraic gossip: A network coding approach to optimal multiple rumor mongering," Allerton'04, Allerton, IL, Sept. 2004.

[4] M.D. Dikaiakos, S. Iqbal, T. Nadeem, and L. Iftode, "Vitp: An information transfer protocol for vehicular computing," VANET'05, Sept. 2005.

[5] M. Guo, M.H. Ammar, and E.W. Zegura, "V3: A vehicle-to-vehicle live video streaming architecture," PerCom'05, March 2005.

[6] T. Ho, M. Médard, R. Koetter, D.R. Karger, M. Effros, J. Shi, and B. Leong, "A random linear network coding approach to multicast," IEEE Trans. Inf. Theory, vol.52, no.10, pp.4413-4430, Oct. 2006.

[7] G. Korkmaz, E. Ekici, F. Özgüner, and U. Özgüner, "Urban multihop broadcast protocols for inter-vehicle communication systems," VANET'04, Philadelphia, PA, USA, Oct. 2004.

[8] H. Lieu, Revised Monograph on Traffic Flow Theory, U.S. Department of Transportation Federal Highway Administration, 2003.

[9] P. Maymounkov, N.J.A. Harvey, and D.S. Lun, "Methods for efficient network coding," Proc. 44th Annual Allerton Conference on Communication, Control, and Computing, 2006.

[10] T. Nadeem, S. Dashtinezhad, C. Liao, and L. Iftode, "Trafficview: Traffic data dissemination using car-to-car communication," ACM Mobile Computing and Communications Review (MC2R), vol.8, no.3, pp.6-19, July 2003.

[11] M.T. Sun, L. Huang, A. Arora, and T.H. Lai, "Reliable MAC layer multicast in IEEE Wireless Networks," ICCP'02, Vancouver, Aug. 2002.

[12] R.M. Yadumurthy, A.C.H., M. Sadashivaiah, and R. Makanaboyina, "Reliable mac broadcast protocol in directional and omni-directional transmissions for vehicular ad hoc networks," VANET'05, Sept. 2005.

[13] W. Yuen, R. Yates, and C. Sung, "Effect of node mobility on highway mobile infostation networks," MSWiM'03, Sept. 2003.

\section{Appendix}

Lemma 2: As $n$ goes to infinity, we have

$$
\lim _{n \rightarrow \infty} \sum_{k=n r}^{n(1+r)} \frac{1}{k}=\ln \left(1+\frac{1}{r}\right)
$$

Proof - Since the summation is a monotonically decreasing function, we can approximate it by integrals:

$$
\begin{aligned}
& \int_{n r}^{n(1+r)+1} \frac{1}{x} d x \leq \sum_{k=n r}^{n(1+r)} \frac{1}{k} \leq \int_{n r-1}^{n(1+r)} \frac{1}{x} d x \\
& \ln \frac{n(1+r)+1}{n r} \leq \sum_{k=n r}^{n(1+r)} \frac{1}{k} \leq \ln \frac{n(1+r)+1}{n r-1}
\end{aligned}
$$

As $n$ goes to infinity, both upper and lower bound become tight, so we have:

$$
\begin{aligned}
\lim _{n \rightarrow \infty} \sum_{k=n r}^{n(1+r)} \frac{1}{k} & =\lim _{n \rightarrow \infty} \ln \frac{n(1+r)}{n r} \\
& =\ln \left(1+\frac{1}{r}\right)
\end{aligned}
$$

